ALEA, Lat. Am. J. Probab. Math. Stat. 16, 1077-1087 (2019)

DOI: 10.30757/ALEA.v16-39

\title{
The upper threshold in ballistic annihilation
}

\section{Debbie Burdinski, Shrey Gupta and Matthew Junge}

Harvard University

Cambridge, MA.

E-mail address: debbie_burdinski@hms.harvard.edu

Duke University

Durham, NC.

E-mail address: shrey.gupta@duke.edu

Bard College

Annandale-on-Hudson, NY.

E-mail address: mjunge@bard.edu

\begin{abstract}
We consider a version of ballistic annihilation with particles placed at the integer points on the real line. Each is independently assigned either speed0 with probability $p$, or speed- \pm 1 symmetrically with the remaining probability. All particles simultaneously begin moving at their assigned speeds and mutually annihilate upon colliding. A renewal property lets us equate survival of a particle to the survival of a Galton-Watson process. An immediate application of our result is an upper bound for the critical probability when particles have unit spacings. This comes from a rigorous, computer-assisted approximation of the Galton-Watson process offspring distribution.
\end{abstract}

\section{Introduction}

Two decades ago, physicists devoted considerable attention to a simple but difficult to analyze process called ballistic annihilation (Elskens and Frisch, 1985; Carnevale et al., 1990; Belitsky and Ferrari, 1995; Droz et al., 1995; Martin and Piasecki, 1994; Krapivsky et al., 1995; Redner, 1997; Trizac, 2002). Particles are placed on the real line according to a unit intensity Poisson point process and each is independently assigned a speed according to a probability measure $\nu$. After this assignment, the model is deterministic; particles move at their speed and mutually annihilate upon colliding. The canonical example is with speeds from $\{-1,0,1\}$ sampled according to the symmetric measure

$$
\nu=\frac{1-p}{2} \delta_{-1}+p \delta_{0}+\frac{1-p}{2} \delta_{1} .
$$

Received by the editors March 23th, 2019; accepted September 25th, 2019.

2010 Mathematics Subject Classification. 60K35.

Key words and phrases. Interacting particle system. 
Physicists refer to this as an $A+A \rightarrow 0$ process. Ballistic annihilation was introduced in Elskens and Frisch (1985) with just two speeds. The goal was to study interactions of ideal gas particles. A few years later, it was discussed in the context of arbitrary continuous $\nu$ (Ben-Naim et al., 1993). The authors' motivation was to understand "intriguing features" in the decay kinetics of irreversible aggregation, $A_{i}+A_{j} \rightarrow A_{i+j}$, which has been used to model coalescence of fluid vortices (McWilliams, 1984) and planet formation by accretion (Weaver and Danly, 1989). The authors gave heuristics for the decay rate of particles, and conjectured it responds continuously to perturbations in the speed measure.

The followup work Krapivsky et al. (1995) predicted more interesting behavior in ballistic annihilation with discrete speeds. It is thought that the process undergoes an abrupt phase transition. Consider ballistic annihilation with the measure from (1.1). We will call speed- 0 particles inert and speed- \pm 1 particles active. Let $\theta_{t}(p)$ be the probability an inert particle survives up to time $t$, and $\theta(p)=\theta_{\infty}(p)$ be the probability it is never annihilated. Krapivsky et al. (1995) inferred that the critical value

$$
p_{c}=\inf \{p: \theta(p)>0\}
$$

is equal to $1 / 4$. They also made precise predictions for the behavior of $\theta_{t}$ :

$$
\theta_{t}(p) \sim\left\{\begin{array}{ll}
C_{p} t^{-1}, & p<p_{c} \\
C t^{-2 / 3}, & p=p_{c} \\
2-p^{-1 / 2}, & p>p_{c}
\end{array}, \text { with } C_{p}=\frac{2 p}{(1-4 p) \pi} \text { and } C=\frac{2^{2 / 3}}{4 \Gamma(2 / 3)^{2}}\right.
$$

A simple heuristic is given in Droz et al. (1995) for why $p_{c}=1 / 4$. Active particles move towards one another at relative speed 2 , while the gap between a moving and inert particle is covered at relative speed 1 . Thus, collisions between active particles ought to occur twice as often as those between inert and active particles. If we look at all of the collisions in a large interval, then each is one of three possibilities:

$$
(0,-1),(1,0) \text {, and }(1,-1) \text {. }
$$

Doubling the $(1,-1)$-collisions to account for the prediction that these occur twice as often, we expect on average that for every eight particles removed, two are inert particles. So, when $p=1 / 4$ the collision types balance.

Symmetry ensures that active particles are annihilated almost surely (see Dygert et al., 2019, Proposition 16.) Note that Krapivsky et al. (1995) also provides a description of the decay density of speed - \pm 1 particles in the different regimes of $p$. For $p<1 / 4$ the right tail is predicted to have exponent -1 . At criticality it is claimed to be $-2 / 3$, and for $p>p_{c}$ they infered that the survival time decays at an exponential rate. The article Droz et al. (1995) addressed these conjectures (including those for $\theta_{t}(p)$ predicted by Krapivsky et al., 1995) by deriving a complicated differential equation involving the distance between particles at time $t$. However, the argument makes a non-rigorous assumption that configurations are independent at different times. Krapivsky et al. (1995) point out in that there is still need for methods "that would provide better intuitive insights into the intriguing qualitative features of ballistic annihilation." 
Remark added in revision. While our paper was in revision, several tremendous advances were made by Haslegrave, Sidoravicius, and Tournier. They effectively solved the case with symmetric speeds in Haslegrave et al. (2018). This includes a proof that $p_{c}=1 / 4$, confirmation of (1.2), and the bounds $.2354<p_{c}^{\prime}<.2406$ (the last bound confirming one of our conjectures). Their results hold for general spacings and establishes universality of the phase transition. Junge and Lyu (2018) generalized parts of Haslegrave et al. (2018) to the asymmetric 3-velocity ballistic annihilation. While the criterium proven in our paper was not used in Haslegrave et al. (2018) nor Junge and Lyu (2018), it may yet find applications in followup work.

Results. In this article, we consider ballistic annihilation with an inert particle, which we will call the seed, at the origin, and particles placed on $\mathbb{Z}^{+}$with i.i.d. speeds sampled according to $\nu$ from (1.1). To distinguish our case from ballistic annihilation with exponentially distributed spacings, we define $\psi(p)$ as the probability the seed is never annihilated. Considering one- rather than two-sided ballistic annihilation does not change whether $\psi(p)$ is positive. Using independence of the process on either side of the seed, we have $\psi(p)$ is the square root of the two-sided survival probability.

Our main result relates the structure of surviving inert particles to a GaltonWatson tree. The idea is that there is a random index $\eta$ for which (a) when restricted to just particles in $[0, \eta]$ only inert particles survive, and (b) the process regenerates at the rightmost surviving particle. If $Z$ is the number of surviving inert particles in the process restricted to $[0, \eta]$, then (b) guarantees that each of these particles will independently spawn $Z$-distributed more surviving inert particles. Because each new generation is determined independently, we obtain a Galton-Watson process that counts surviving particles. Using the standard criteria for survival of a GaltonWatson process, we obtain our main result.

Theorem 1.1. Let $Z$ be as in Proposition 2.4. It holds that $\psi(p)>0$ if and only if $\mathbf{E} Z>1$.

Note that we suppress the $p$-dependence in the expectation and write $\mathbf{E} Z$ in place of $\mathbf{E}_{p} Z$. In 1082 2.5, we explain how to adapt Theorem 1.1 to the usual setting with exponentially-distributed spacings. Thus, a similar equivalence holds for $\theta(p)$. The resulting Galton-Watson tree encodes the dependence structure between inert particles in a new way. In the construction of $Z$, we see that the main contribution to the survival of inert particles comes from what happens in a finite window to the right of where a 1-particle annihilates an inert particle. This provides a connection between the lifespans of inert and active particles. We may be able to control $\mathbf{E} Z$ by understanding the survival time of active particles. For example, proving $\psi(p)=0$ implies active particles have infinite expected lifetimes may be a useful step in a proof by contradiction.

There has also been interest in proving upper bounds on $p_{c}$. Our result has an application for this, but first we describe the history. Dygert et al. (2019) proved that $p_{c} \leq .3313$. Additionally, Sidoravicius and Tournier (2017) considered ballistic annihilation. Sidoravicius and Tournier proved that $p_{c} \leq 1 / 3$, and outline an approach to prove $p_{c} \leq 0.3280$. Their proof, like the one contained in this work, is recursive in nature. The configuration of particle speeds is revealed in blocks, and the number of surviving particles can be estimated by the sub-configuration 
in these blocks. The main difference is that the renewal time in Proposition 2.4 leads to a particularly useful Galton-Watson process. We are not trying to start a hunt for bounds closer and closer to the conjectured value. Rather, by improving the known bound, we reveal a new perspective into what promotes survival of inert particles.

For ballistic annihilation with unit spacings, triple collisions may slightly change the critical threshold. Define the critical probability as

$$
p_{c}^{\prime}=\inf \{p: \psi(p)>0\} .
$$

Let $q=(1-p) / 2$, so that the probability three consecutive particles triple collide is $p q^{2}$ (i.e. a $(1,0,-1)$ configuration). This is $\approx .07$ when $p=1 / 4$. With exponential spacings, the process loses one inert and one active particle whenever this configuration occurs. However, with the triple collision, an extra active particle is destroyed. The heuristic at (1.3) predicts that, after one time unit, the density of 0 -particles is

$$
w=p-2 p q+p q^{2} .
$$

The $2 p q$ term accounts for the configurations $(1,0)$ and $(0,-1)$. We add $p q^{2}$ to prevent double counting triple collisions. Similarly, the density of active particles is

$$
z=2\left(q-q^{2}-p q\right)
$$

The heuristic at (1.3) suggests we solve $w=\frac{1}{4}(z+2 w)$, which yields $p \approx .2450$. Accordingly, we conjecture that $p_{c}^{\prime}<.2450$. Note that this was confirmed by Haslegrave et al. (2018).

Understanding survival of inert particles appears to be equally interesting and challenging whether the spacings are deterministic, or Exponential(1) distributed. This is supported by recent findings (Broutin and Marckert, 2017) for the closely related bullet process with finitely many particles and a non-atomic probability measure on speeds. They find that the law for the number of surviving particles is independent of the initial spacings. An immediate benefit of our approach is an upper bound on $p_{c}^{\prime}$.

Proposition 1.2. $p_{c}^{\prime} \leq .2870$.

We obtain this estimate from Theorem 1.1 and a lower bound that gives $\mathbf{E} Z>1$ when $p>.2870$. The lower bound comes from considering certain random times before $\eta$ at which we can stochastically lower bound $Z$. We then use a computer to estimate these probabilities. This is completely rigorous, but requires too many calculations to be done by hand.

Notation. We call particles with speed- \pm 1 active. When the speed is relevant, we call them \pm 1 -particles. The randomness in ballistic annihilation is an initial vector of particle speeds $\mathbf{X}=\left(X_{i}\right)_{i=0}^{\infty}$ with $X_{0}=0$ and the $X_{i}$ for $i \geq 1$ are i.i.d. with law $\nu$ from (1.1). Let $\mathbf{X}[i, j]=\left(X_{i}, \ldots, X_{j}\right)$ be the restriction to the coordinates between $i \leq j$.

Let $a_{i}$ denote the particle initially at $i$. We will let $a_{i} \leftrightarrow a_{j}$ mean that particles at $i$ and $j$ mutually annihilate. We will sometimes use the more specific notation $a_{i} \mapsto a_{j}$ for when the active particle from $i$ annihilates the inert particle at $j$. Given $\mathbf{x}=\left(x_{i}, x_{i+1}, \ldots, x_{j}\right)$, we define $\mathrm{BA}(\mathbf{x})$ to be ballistic annihilation on $\mathbb{R}$ with particles at $i, i+1, \ldots, j$ where the particle at $k$ has speed $x_{k}$. Run $\mathrm{BA}(\mathbf{x})$ until 
every collision that could occur has occurred (this takes at most $j-i$ time units). Let $\xi(\mathbf{x})=\left(\xi_{i}, \ldots, \xi_{j}\right)$ with

$$
\xi_{k}(\mathbf{x})=x_{k} \mathbf{1}\left\{a_{k} \text { survives }\right\}+2 \cdot \mathbf{1}\left\{a_{k} \text { is annihilated }\right\} .
$$

This records the speeds of surviving particles from $\mathrm{BA}(\mathbf{x})$ and labels annihilated particles with a 2 . For example if $\mathbf{x}=(0,1,-1,1)$ then $\xi(\mathbf{x})=(0,2,2,1)$.

\section{An embedded Galton-Watson process}

To show that inert particles survive with positive probability, it suffices to establish that the seed is never annihilated with positive probability. This is because an inert particle, say $a_{n}$, has $n$ particles to its left, and thus has some positive probability of not being annihilated from that side. For example, all left particles are inert with probability $p^{n-1}$. Conditional on surviving from the left, the probability that $n$ is never annihilated by a particle from the right is the same as the probability the seed survives. This follows via the coupling that aligns the speeds to the right of the seed with those to the right of $a_{n}$ in two independent processes.

The construction of a Galton-Watson process hinges upon a renewal structure that starts with two simple observations about the range of dependence caused by collisions.

Lemma 2.1. Let $i<j$ and condition that $X_{j}=-1$. The random variables $\left(X_{k}\right)_{k>j}$ are $\nu$-distributed and independent of the event $\left\{a_{i} \leftrightarrow a_{j}\right\}$.

Proof: The particles to the right of $a_{j}$ cannot influence the event $\left\{a_{i} \leftrightarrow a_{j}\right\}$, thus the speeds are independent of the event.

If an active particle destroys an inert particle, then this induces a short range dependence. We know that all of the active particles that could reach the inert particle before its destroyer arrives must be annihilated. However, if we look sufficiently far away the particle speeds are once again independent.

Lemma 2.2. Let $i<j$ and condition that $X_{j}=0$. The random variables $\left(X_{k}\right)_{k>j+(j-i)}$ are independent of the event $\left\{a_{i} \mapsto a_{j}\right\}$.

Proof: It is elementary to work out that the particles beyond $j+(j-i)$ cannot reach $a_{j}$ before $a_{i}$ does, thus their speeds are independent of the event.

Remark 2.3. The speed $X_{j+(j-i)}$ is independent of $\left\{a_{i} \mapsto a_{j}\right\}$, but possibly $a_{j+(j-i)}$ triple collides with $a_{j}$.

For the event in Lemma 2.1, we call $[i, j]$ the window of dependence, because particle speeds with indices beyond $j$ are independent of $\left\{a_{i} \leftrightarrow a_{j}\right\}$. For Lemma 2.2, the window of dependence is $[i, 2 j-i]$. We obtain a renewal by looking out to the first time the window of dependence stops growing.

Proposition 2.4. There is a random index $\eta$ with $Z$ surviving inert particles and no surviving active particles in $\mathrm{BA}(\mathbf{X}[0, \eta])$. $\eta$ and $Z$ almost surely satisfy one of the following three conditions:

(i) $\eta=\infty, Z \geq 1$.

(ii) $\eta=1, Z=0$. 
(iii) $1 \leq \eta, Z<\infty$. Let $\kappa$ be the index of the rightmost particle counted by $Z$. There exist independent copies $\eta^{\prime}$ and $Z^{\prime}$ of $\eta$ and $Z$ such that there are no surviving active particles, and $Z^{\prime}$ surviving inert particles in $\mathrm{BA}(\mathbf{X}[\kappa, \eta+$ $\left.\left.\eta^{\prime}\right]\right)$. Moreover, the speeds $\left(X_{i}\right)_{i>\eta+\eta^{\prime}}$ are i.i.d. $\nu$-distributed.

Proof: We define $\eta$ via an algorithm that constructs a non-decreasing sequence $\left(\eta_{t}\right)$ of integers that starts with $\eta_{0}=1$. Subsequent terms are defined recursively in the following way.

(I) If $X_{1} \in\{-1,0\}$, then set $\eta=1$.

(II) If $X_{1}=1$, then $\eta_{t+1} \geq \eta_{t}$ is given by one of the following:

(II.a) If there is a surviving 1-particle in $\mathrm{BA}\left(\mathbf{X}\left[1, \eta_{t}\right]\right)$, then let $\tau_{t}$ be the index of the left-most one, and let $\eta_{t+1}>\eta_{t}$ be such that the particle started at $\tau_{t}$ collides with the one started at $\eta_{t+1}$.

(II.b) If there is no surviving 1-particle in $\mathrm{BA}\left(\mathbf{X}\left[1, \eta_{t}\right]\right)$, then let $\eta_{t+1}$ be the rightmost value in the union of all windows of dependence induced by the collisions in $\mathrm{BA}\left(\mathbf{X}\left[1, \eta_{t}\right]\right)$.

(III) If $\eta_{t}=\eta_{t+1}$, then halt the algorithm and set $\eta=\eta_{t}$.

(IV) If the algorithm never terminates, then set $\eta=\sup \eta_{t}=\infty$.

We start by considering the three simplest cases. If $\eta=1$ and $X_{1}=-1$, then (ii) occurs. If instead $X_{1}=0$, then (iii) is satisfied, since we can view the inert particle at 1 as the new seed in the process shifted right by 1 . If $\eta=\infty$, then Case (I) did not occur and the seed must survive. Thus, (i) holds.

To have $1<\eta<\infty$ requires that Case (II.b) occurs. This ensures that the seed is not destroyed in $\operatorname{BA}(\mathbf{X}[0, \eta])$ and the only surviving particles are inert. Let $\kappa$ be the index of the rightmost such particle. Since $a_{\kappa}$ survives in $\mathrm{BA}(\mathbf{X}[0, \eta])$, all of the particles started in $[\kappa+1, \eta]$ mutually annihilate. By Lemma 2.1 and Lemma 2.2 this mutual annihilation does not influence the speeds of particles started beyond $\eta$. To obtain $\eta^{\prime}$ and $Z^{\prime}$, imagine the particle at $\kappa$ is at $\eta$ and repeat the algorithm shifted by $\eta$. Since only a -1 -particle can destroy $a_{\kappa}$, the presence of a gap $\eta-\kappa$ does not change the way we obtain $\eta^{\prime}$ and $Z^{\prime}$. Both are independent and have the claimed distribution because the window of dependence is $[1, \eta]$ and so the speeds of particles started beyond $\eta$ are i.i.d. $\nu$-distributed,

Remark 2.5. A similar statement holds when particles are placed according to a unit intensity Poisson process. Lemma 2.1 and Lemma 2.2 still hold in this setting, and thus we can follow the same steps to obtain a renewal as in Proposition 2.4.

Because the process renews after $\eta$ we can link the expected number of surviving inert particles in $\mathrm{BA}(\mathbf{X}[0, \eta])$ to $\psi(p)$ via a Galton-Watson process.

Proof of Theorem 1.1: If $\psi(p)>0$, then (i) happens with probability $\psi(p)$. It follows from the Birkhoff ergodic theorem that, on the event the seed survives, there are almost surely infinitely many surviving inert particles in $\mathrm{BA}(\mathbf{X})$. Thus, $P(Z=\infty)=\psi(p)$, and $\mathbf{E} Z=\infty$.

Next, suppose that $\mathbf{E} Z>1$. Let $\left(\eta_{i}, Z_{i}\right)_{i \geq 0}$ be i.i.d. copies of $\eta$ and $Z$, respectively. By Proposition 2.4, $\mathrm{BA}(\mathbf{X}[0, \eta])$ has $Z_{0}$ surviving inert particles. We consider three cases:

- If $Z_{0}=0$, then the process terminates. If not, then (iii) ensures that the rightmost surviving particle at $\kappa_{1}$ counted by $Z_{0}$ will spawn $Z_{1}$ more surviving inert particles in $\mathrm{BA}\left(\mathbf{X}\left[\kappa_{1}, \eta_{0}+\eta_{1}\right]\right)$. 
- If $Z_{1}=0$, then $a_{\kappa_{1}}$ is destroyed. We then consider the second rightmost particle at $\kappa_{2}$ counted by $Z_{0}$. By repeating the construction in Proposition 2.4 we obtain $Z_{2}$ more inert particles in $\mathrm{BA}\left(\mathbf{X}\left[\kappa_{2}, \eta_{0}+\eta_{1}+\eta_{2}\right]\right)$. We then repeat this procedure from the rightmost particle counted by $Z_{2}$.

- If $Z_{1}>0$, then we repeat this procedure again from the rightmost surviving particle.

Call the $Z_{i}$ inert particles counted at each step the children of whatever inert particle was being considered. Because the $\left(Z_{i}\right)$ are i.i.d., this results in a Galton-Watson process. Since we always consider the rightmost surviving inert particle, we explore the corresponding tree in a depth-first manner. The assumption $\mathbf{E} Z>1$ ensures there is a positive probability that a path to infinity is discovered and so the seed is never annihilated.

Remark 2.6. Each unexplored site on the infinite path from Theorem 1.1 corresponds to an inert particle that survives for all time in $\mathrm{BA}(\mathbf{X})$. By keeping track of the values $Z_{i}$ and $\kappa_{i}$, this tree encodes the set of all surviving particles.

\section{Approximating $\mathbf{E} Z$}

The recursive definition of $\eta$ from Proposition 2.4 suggests it would be difficult to calculate $\mathbf{E} Z$ explicitly. However, there are certain indices before $\eta$ at which we can obtain lower bounds on $\mathbf{E} Z$. It helps to explain in stages. First, we consider the effect of $a_{1}$.

3.1. The effect of $\mathrm{BA}(\mathbf{X}[0,1])$. The simplest lower bound on $Z$ is to look at what happens in $\operatorname{BA}(\mathbf{X}[0,1])$. If $X_{1}=-1$, then $Z=0$, and if $X_{1}=0$, then $Z=2$. This gives

$$
Z \succeq \mathbf{1}\left\{X_{1}=0\right\} 2 .
$$

Thus, if $p>1 / 2$, we have $\mathbf{E} Z>1$. Equivalently, $p_{c}^{\prime} \leq 1 / 2$. This is a start, but not so interesting. The same statement could be proven by accounting for the number of inert particles versus active particles with a $p$-biased random walk. Survival of the seed is equivalent to the walk never returning to 0 , which happens with positive probability when $p>1 / 2$.

Let us go one step further by considering the case $\eta>1$. If $X_{1}=1$, then the construction of $\eta$ ensures that the seed survives in $\mathrm{BA}(\mathbf{X}[0, \eta])$. Thus, $Z \succeq 1$ on this event. This gives the more meaningful bound

$$
Z \succeq \mathbf{1}\left\{X_{1}=0\right\} 2+\mathbf{1}\left\{X_{1}=1\right\} .
$$

Taking expectation in (3.2) yields $\mathbf{E} Z \geq 2 p+\frac{1-p}{2}$. When $p>1 / 3$, this is larger than 1 . Thus, we arrive easily at the bound $p_{c}^{\prime} \leq 1 / 3$, which is proven in Dygert et al. (2019) and proven for $p_{c}$ in Sidoravicius and Tournier (2017).

3.2. The effect of $a_{\gamma_{1}}$. We can further extract some benefit from the 1-particle at 1. Let $\gamma_{1}$ be the index of the particle that destroys $a_{1}$. Let $Y$ be 1 plus the number of surviving inert particles in the window of dependence induced by the event $\left\{a_{1} \leftrightarrow a_{\gamma_{1}}\right\}$. The plus 1 is to count the seed. Depending on whether $X_{\gamma_{1}}=-1$ or 0 , this window is either $\left[1, \gamma_{1}\right]$ or $\left[1,2 \gamma_{1}-1\right]$. 


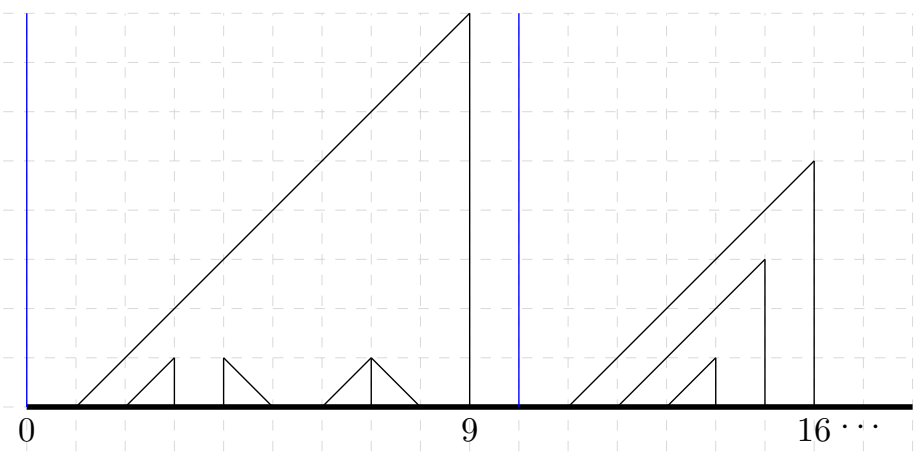

Figure 3.1. In this example, $\gamma_{1}=9$. We are left with $Y=2$ surviving inert particles in $\mathrm{BA}\left(\mathbf{X}\left[0,2 \gamma_{1}-1\right]\right)$.

If the window of dependence is $\left[1,2 \gamma_{1}-1\right]$, then the surviving particles in $\mathrm{BA}\left(\mathbf{X}\left[1,2 \gamma_{1}-1\right]\right)$ will consist of an uninterrupted sequence of surviving inert particles, and to their right an uninterrupted sequence of surviving 1-particles. The process will renew once all of the surviving 1-particles have been dealt with by the algorithm in Proposition 2.4. Thus, the inert particles that survive in $\operatorname{BA}\left(\mathbf{X}\left[1,2 \gamma_{1}-1\right]\right)$ also survive in $\mathrm{BA}(\mathbf{X}[0, \eta])$. It follows that $\mathbf{1}\left\{X_{1}=1\right\} Y \preceq \mathbf{1}\left\{X_{1}=1\right\} Z$. This lets us improve (3.2) to the following dominance relation

$$
Z \succeq \mathbf{1}\left\{X_{1}=0\right\} 2+\mathbf{1}\left\{X_{1}=1\right\} Y .
$$

Figure 3.1 depicts a realization in which $Y=2$. To better understand $Y$, we decompose it relative to the behavior of $\gamma_{1}$ :

$$
\begin{aligned}
Y & =\sum_{n=2}^{\infty} \mathbf{1}\left\{\gamma_{1}=n\right\} Y \\
& =\sum_{n=2}^{\infty} \mathbf{1}\left\{X_{\gamma_{1}}=-1, \gamma_{1}=n\right\}+\mathbf{1}\left\{X_{\gamma_{1}}=0, \gamma_{1}=n\right\} Y \\
& =1+\sum_{n=2}^{\infty} \mathbf{1}\left\{X_{\gamma_{1}}=0, \gamma_{1}=n\right\}(Y-1) .
\end{aligned}
$$

Note that $\gamma_{1} \geq 2$ since we only introduce $\gamma_{1}$ when $X_{1}=1$, and so the particle that destroys $a_{1}$ must start at 2 or beyond. All of the cases where $X_{\gamma_{1}}=-1$ are included in the leading 1 summand, so we devote our attention to when $X_{\gamma_{1}}=0$. Let

$$
A_{n}=\left\{X_{\gamma_{1}}=0, \gamma_{1}=n\right\} \subseteq\{0\} \times\{1\} \times\{-1,0,1\}^{2 n-2}
$$

be the set of sub-configurations for which $\gamma_{1}=n$ and $X_{\gamma_{1}}=0$. Notice we only need to know the entries up to $2 n-1$ to determine if $a_{1} \mapsto a_{n}$.

In light of (3.4), we would like to understand $\mathbf{E}\left[\mathbf{1}\left\{A_{n}\right\}(Y-1)\right]$. An important observation is that $Y-1 \geq 0$ since the seed always survives in $\mathrm{BA}\left(\left[0,2 \gamma_{1}-1\right]\right)$. This means we can lower bound the expectation by computing its value for finitely many $n$. We do so by counting surviving inert particles in the final state of $\operatorname{BA}\left(\mathbf{X}\left[0,2 \gamma_{1}-\right.\right.$ 1]) in each of the $3^{2 n-2}$ possible realizations of particle speeds from $\{0\} \times\{1\} \times$ $\{-1,0,1\}^{2 n-2}$. 
Given $\mathbf{x} \in A_{n}$, let $I(\mathbf{x})$ be the number of 0-entries in $\mathbf{x}$. By the definition of $\nu$ at (1.1), the probability $\mathbf{x}$ occurs is

$$
q_{n}(\mathbf{x}):=p^{I(\mathbf{x})}\left(\frac{1-p}{2}\right)^{2 n-2-I(\mathbf{x})} .
$$

Letting $Y(\mathbf{x})$ be the number of surviving inert particles in $\mathrm{BA}(\mathbf{x})$, we then have

$$
\mathbf{E}\left[\mathbf{1}\left\{A_{n}\right\}(Y-1)\right]=\sum_{\mathbf{x} \in A_{n}} q_{n}(\mathbf{x})[Y(\mathbf{x})-1] .
$$

This is possible for a computer to calculate for small values of $n$. For instance, if we compute for $n \leq 18$ (approximately 400 million cases) and take expectation in (3.4), we obtain the bound

$$
\mathbf{E} Y \geq 1+\sum_{n=2}^{18} \sum_{x \in A_{n}} q_{n}(\mathbf{x})[Y(\mathbf{x})-1] .
$$

Call the quantity on the right in (3.7) $m(p)$. By (3.3) we have

$$
\mathbf{E} Z \geq 2 p+\frac{1-p}{2} m(p) .
$$

By numerically checking the boundary values of $p$, we find that $\mathbf{E} Z>1$ when $p>.2914$. Note that $m(.2914) \approx 1.1178$. So the "gain" we had from the previous calculation is $\approx .1178$ more expected surviving inert particles.

3.3. Using a surviving 1-particles from $\mathrm{BA}\left(\mathbf{X}\left[0,2 \gamma_{1}-1\right]\right)$. A simple way to optimize further is by re-using the previous calculation for configurations from $A_{n}$ in which there is a single surviving 1-particle at $2 n-1$, and otherwise only inert particles.

Formally, let

$$
A_{n}^{\prime}=\left\{\mathbf{x} \in A_{n}: \xi_{2 n-1}(\mathbf{x})=1, \xi_{i}(\mathbf{x}) \in\{0,2\} \text { for } 0 \leq i \leq 2 n-2\right\}
$$

be the set of all such configurations. Recall that $\xi$ is defined at (1.4). We claim that each $\mathbf{x}^{\prime} \in A_{n}^{\prime}$ provides an independent $Y-1$ distributed number of inert particles. This is because the particle speeds to the right of $a_{2 \gamma_{1}-1}$ are independent. Thus, we can couple the number of surviving inert particles induced by $a_{2 \gamma_{1}-1}$ to $Y-1$. We subtract 1 so we do not double count the seed. Let $b(p)=\sum_{n=1}^{18} \sum_{\mathbf{x}^{\prime} \in A_{n}^{\prime}} q_{n}\left(\mathbf{x}^{\prime}\right)$ be the probability of a configuration from $A_{n}^{\prime}$. Each time this occurs we obtain an expected $m(p)-1$ more inert particles with $m(p)$ from (3.7). This will happen geometric $(b(p))$-distributed many times, which has expectation $b(p) /(1-b(p))$. It follows that we can improve our bound from (3.8) to the following

$$
\mathbf{E} Z \geq 2 p+\frac{1-p}{2}\left(m(p)+\frac{b(p)}{1-b(p)}(m(p)-1)\right) .
$$

It takes a computer about three hours to obtain an algebraic expression for $b(p)$. After doing so, we find that $\mathbf{E} Z>1$ when $p>.2870$. In this case we have $m(p) \approx$ 1.1713 and $b(p) \approx .1226$. 
3.4. Further benefit from surviving active particles. The bound we obtain on $p_{c}^{\prime}$ is as far as seemed reasonable to push our technique. More complicated calculations can be done where one considers the effect of other configurations of surviving 1particles to the right of $\gamma_{1}$. For example, one might consider the case that there is a single surviving 1 -particle at $2 \gamma_{1}-k$ with $k \geq 1$ (we only consider $k=1$ ). However, we found that the improvements to our bound were very small, trimming about .004 from our bound on $p_{c}$, and did not justify the added complexity to the argument. It is our belief that the main benefit to estimating $\mathbf{E} Z$ would come out of extending our approach and looking out to distances $n>18$. When $p=.2870$ we exactly compute $P\left(\gamma_{1} \leq 18\right)=.9018$. So, we are missing about $1 / 10$ of the right tail, which ought to contain a significant number of surviving inert particles. However, without a clever insight, extending much further appears computationally intractable.

Acknowledgments. Thanks to Laurent Tournier and Rick Durrett for helpful suggestions. We also appreciate the careful readings and useful comments from the anonymous referees.

\section{References}

V. Belitsky and P. A. Ferrari. Ballistic annihilation and deterministic surface growth. J. Statist. Phys. 80 (3-4), 517-543 (1995). MR1342240.

E. Ben-Naim, S. Redner and F. Leyvraz. Decay kinetics of ballistic annihilation. Phys. Rev. Lett. 70, 1890-1893 (1993). DOI: 10.1103/PhysRevLett.70.1890.

N. Broutin and J.-F. Marckert. The combinatorics of the colliding bullets problem. ArXiv Mathematics e-prints (2017). arXiv: 1709.00789.

G. F. Carnevale, Y. Pomeau and W. R. Young. Statistics of ballistic agglomeration. Phys. Rev. Lett. 64, 2913-2916 (1990). DOI: 10.1103/PhysRevLett.64.2913.

M. Droz, P.-A. Rey, L. Frachebourg and J. Piasecki. Ballistic-annihilation kinetics for a multivelocity one-dimensional ideal gas. Phys. Rev. E 51, 5541-5548 (1995). doi:10.1103/PhysRevE.51.5541. DOI: 10.1103/PhysRevE.51.5541.

B. Dygert, C. Kinzel, M. Junge, A. Raymond, E. Slivken and J. Zhu. The bullet problem with discrete speeds. Electron. Commun. Probab. 24, Paper No. 27, 11 (2019). MR3962477.

Y. Elskens and H. L. Frisch. Annihilation kinetics in the one-dimensional ideal gas. Phys. Rev. A 31, 3812-3816 (1985). DOI: 10.1103/PhysRevA.31.3812.

J. Haslegrave, V. Sidoravicius and L. Tournier. The three-speed ballistic annihilation threshold is 1/4. ArXiv Mathematics e-prints (2018). arXiv: 1808.10731.

M. Junge and H. Lyu. The phase structure of asymmetric ballistic annihilation. ArXiv Mathematics e-prints (2018). arXiv: 1811.08378.

P. L. Krapivsky, S. Redner and F. Leyvraz. Ballistic annihilation kinetics: The case of discrete velocity distributions. Phys. Rev. E 51, 3977-3987 (1995). DOI: 10.1103/PhysRevE.51.3977.

P. A. Martin and J. Piasecki. One-dimensional ballistic aggregation: Rigorous long-time estimates. Journal of Statistical Physics 76 (1), 447-476 (1994). DOI: 10.1007/BF02188670.

J. C. McWilliams. The emergence of isolated, coherent vortices in turbulent flow. AIP Conference Proceedings 106 (1), 205-221 (1984). DOI: 10.1063/1.34273. 
Sidney Redner. Scaling theories of diffusion-controlled and ballistically controlled bimolecular reactions, pages 3-28. Cambridge University Press (1997). DOI: 10.1017/CBO9780511564284.003.

V. Sidoravicius and L. Tournier. Note on a one-dimensional system of annihilating particles. Electron. Commun. Probab. 22, Paper No. 59, 9 (2017). MR3718709.

E. Trizac. Scaling in ballistic annihilation kinetics*. Journal of Physics: Condensed Matter 14 (9), 2159-2166 (2002). DOI: 10.1088/0953-8984/14/9/305.

H. Weaver and L. Danly. The formation and evolution of planetary systems (1989). 